\title{
Upregulation and inhibition of the nuclear translocation of Oct4 during multistep gastric carcinogenesis
}

\author{
FATHYIA Y. AL-MARZOQEE ${ }^{1}$, GHALIA KHODER ${ }^{1,5}$, HAIFA AL-AWADHI $^{1}$, RONY JOHN ${ }^{1}$, \\ AZAM BEG $^{2}$, ARON VINCZE ${ }^{3,6}$, FRANK BRANICKI ${ }^{4}$ and SHERIF M. KARAM ${ }^{1}$ \\ ${ }^{1}$ Department of Anatomy, Faculty of Medicine and Health Sciences; ${ }^{2}$ College of Information Technology, \\ UAE University; ${ }^{3}$ Gastroenterology Unit, Tawam Hospital; ${ }^{4}$ Department of Surgery, \\ Faculty of Medicine and Health Sciences, UAE University, Al-Ain, United Arab Emirates
}

Received February 10, 2012; Accepted March 19, 2012

DOI: 10.3892/ijo.2012.1608

\begin{abstract}
Gastric cancer is the fourth most commonly diagnosed malignancy and the second leading cause of cancer-related mortality worldwide. Recent research suggests that tissue stem cells and the self renewal transcription factor, octamer-binding transcription factor 4 (Oct4), could be involved in the development of certain tumors. The aim of this study was to investigate the expression pattern of Oct 4 in normal human stomach and during multistep gastric carcinogenesis. Pyloric antral mucosal tissues were obtained from consenting individuals undergoing endoscopy (due to upper gastrointestinal symptoms) and gastrectomy (due to pyloric antral adenocarcinoma). Some tissue samples were processed to assemble an array of tissue sections representing multistep carcinogenesis and probed using anti-Oct 4 antibodies and lectins specific for $\alpha$-L-fucose or $N$-acetyl-D-glucosamine. Some tissue samples were processed for subcellular fractionation and western blot analysis using the same antibodies. The results revealed that Oct4-expressing cells were found in the proliferative cell compartment of the pit-gland units of microscopically normal gastric mucosal biopsies. Mucosal tissues with evidence of severe gastritis, metaplastic/dysplastic transformation and gastric cancer showed a significant increase in the expression of Oct4 (the labeled area increased from $2 \%$ in the control to 6 and $16 \%$ in the gastritis and cancerous tissues, respectively), suggesting a role for Oct 4 in the early stages of cancer development. Furthermore, the data revealed an alteration in the subcellular distribution of Oct4, possibly due to the inhibition
\end{abstract}

Correspondence to: Dr Sherif M. Karam, Department of Anatomy, Faculty of Medicine and Health Sciences, UAE University, P.O. Box 17666, Al-Ain, United Arab Emirates

E-mail: skaram@uaeu.ac.ae

Present addresses: ${ }^{5}$ College of Pharmacy, Al-Ain University of Science and Technology, Al-Ain, United Arab Emirates; ${ }^{6}$ Department of Internal Medicine, Faculty of Medicine, University of Pécs, Pécs, Hungary

Key words: stomach, gastric cancer, gastric stem cell, gastritis, octamer-binding transcription factor 4 of cytoplasm-to-nucleus translocation during carcinogenesis. In conclusion, this study demonstrates an alteration in the expression pattern and nuclear translocation of Oct 4 during gastric carcinogenesis and may be helpful in designing new modalities for the early detection and/or therapy of gastric cancer.

\section{Introduction}

Although the incidence and mortality rates attributable to gastric cancer have recently been declining, it remains the fourth most common cancer and the second leading cause of cancerrelated mortality worldwide (1). Data from the National Cancer Registry at Tawam Hospital and the Center of Arab Genomic Studies in the United Arab Emirates have shown that gastric cancer is more common than previously believed and that there is an increasing trend in its incidence locally. A greater understanding of gastric carcinogenesis may lead to the design of new modalities for its early detection and prevention (2).

The majority of gastric neoplasms $(95 \%)$ are adenocarcinomas which develop more commonly in the pyloric antrum and are usually of the differentiated intestinal type (3). The pathogenesis of intestinal adenocarcinoma has not yet been fully elucidated. It is usually associated with Helicobacter pylori (H. pylori) infection and is preceded by prolonged pre-cancerous changes which progress through a number of sequential steps: superficial mild gastritis, atrophic gastritis, intestinal metaplasia, dysplasia and finally, invasive carcinoma (4). In addition, previous studies using animal models demonstrating the early stages of gastric cancer development have suggested a role for local (gastric) or distant (bone marrow) stem/progenitor cells in carcinogenesis $(5,6)$.

Limited data are available concerning pyloric antral stem/ progenitor cells in humans and rodents. Previous studies aimed at identifying these cells, analyzing their dynamics and following their fate in mice, have demonstrated their location at the pit-gland junction (isthmus region; Fig. 1) of the epithelial units and their differentiation pathways with a bidirectional migration mode $(7,8)$. However, a recent functional genetic analysis demonstrated the presence of a pluripotent stem cell, not in the isthmus, but at the bottom of the antral glands, which is capable of populating the whole epithelium of the pit-gland unit, implying a unidirectional mode of migration (9). 
Regardless of their location and direction of migration of their progeny, it is not yet known whether these stem/progenitor cells play a role in the development of gastric cancer in humans. Experimental studies in mice have recently provided growing evidence suggesting that gastric epithelial stem/progenitor cells are involved in carcinogenesis (6). For example, a deficiency in trefoil factor 1 , known to control the commitment program of gastric progenitors (10), has been associated with the gradual amplification of these progenitors leading to the development of gastric adenoma and carcinoma (11). Recently, in a previous study, we examined gastric biopsies showing mild-to-severe chronic gastritis, as well as gastric cancer tissues obtained from the safe resection margin and tumor edge, and we observed an amplification of gastric epithelial progenitors in pre-cancerous tissues (12). These data support the long-standing postulate that cancer arises from mutated adult stem cells $(13,14)$.

A number of molecules are involved in the signaling pathways for maintaining the proliferation and differentiation of normal gastrointestinal stem cells (15). The octamer-binding transcription factor 4 (Oct4) is a transcription factor which belongs to the POU family of proteins and binds octamer DNA motifs in the promoters of several genes to regulate the pluripotency of embryonic stem cells (16). Further studies have shown that Oct4 expression is not restricted to the embryonic stem cells (17-20).

The ectopic expression of Oct4 in mice inhibits the differentiation of progenitor cells and promotes epithelial dysplasia, suggesting a role for Oct4 in tumor induction (21). Indeed, the expression of Oct 4 has been demonstrated in tumors of various origin (22-25). Oct4 is also expressed in a number of cell lines established from tumors of the cervix, colon, liver, mammary gland and pancreas (20). The expression of the Oct4 gene in embryonic stem cells, tissue stem cells, and a tumor arising in these tissues, suggests a critical role for Oct4 in maintaining not only the pluripotency of embryonic stem cells, but also the homeostasis of somatic stem cells, as well as the possible role of these cells in the tumorigenesis and tumorigenenicity of cancer.

This study was conducted to determine whether Oct 4 is expressed in the microscopically normal antrum of the human stomach and, consequently, to examine whether there is an alteration in the expression pattern of Oct4 during the multistep process of gastric carcinogenesis.

\section{Materials and methods}

The design and protocols of this study were approved by the Ethics Committees for Research on Human Subjects in the Faculty of Medicine and Health Sciences, UAE University, Al-Ain, United Arab Emirates.

Human tissues. In the present study, gastric mucosal tissues were obtained from the pyloric antrum of patients at Tawam Hospital undergoing endoscopy $(n=89)$ for the investigation of recurrent upper gastrointestinal symptoms, or gastrectomy $(n=3)$ for adenocarcinoma. All patients gave written informed consent prior to the study. The patients were of both genders and aged 20-90 years. Following the endoscopic or surgical procedures, biopsies or cancer tissues (taken from 3 regions: tumor center, tumor edge and from the safe resection margin)

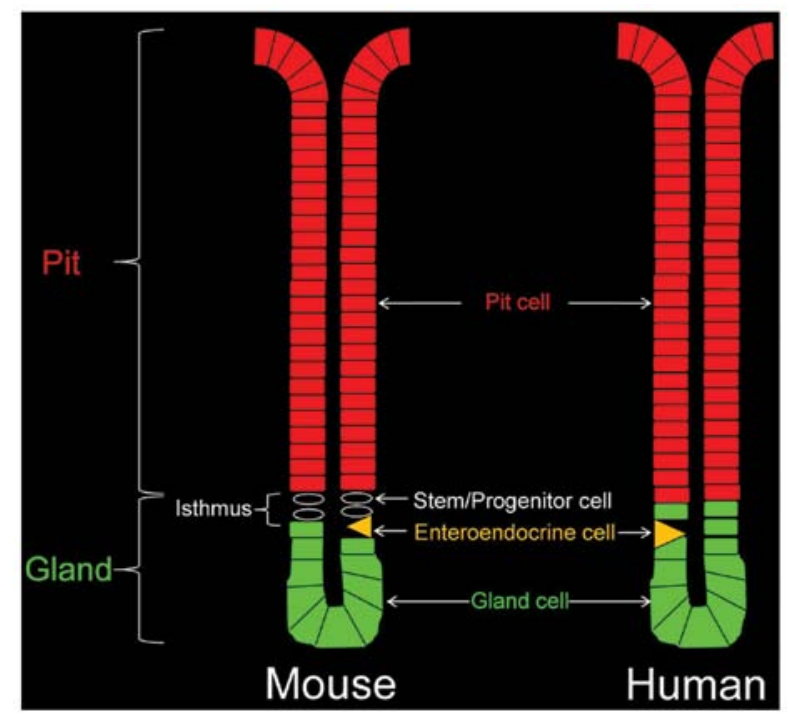

Figure 1. Diagrammatic representation of the epithelial pit-gland units in the pyloric antrum of the mouse and human stomachs. Note that an isthmus region containing granule-free stem/progenitor cells is evident in the mouse unit, but not in the human one.

were immediately processed for immunohistochemistry and protein analyses.

Immunohistochemical studies. Tissues with the dimension of approximately $3 \times 3 \mathrm{~mm}$ (biopsy) or $10 \times 5 \mathrm{~mm}$ (cancer) were immediately immersed overnight in Bouin's solution and then processed for paraffin embedding. Tissue sections were stained with hematoxylin and periodic acid Schiff (PAS) and examined with an Olympus microscope. To categorize gastric mucosal biopsies, the updated Sydney system was utilized (26). Some tissue sections of biopsies representing normal mucosa, as well as mild and severe gastritis were mounted on the same slide along with the cancerous tissues from the safe margin, tumor edge and tumor center.

The tissue sections were deparaffinized, rehydrated and washed in phosphate-buffered saline (PBS). To inhibit endogenous peroxidase activity, the sections were incubated in $3 \%$ hydrogen peroxide in methanol for $1 \mathrm{~h}$. To ensure equal conditions on all biopsy and cancer tissue sections mounted on the same slide, they were circled with a hydrophobic film using a PAP pen (Dako, Glostrup, Denmark). To block non-specific binding, the sections were incubated in $1 \%$ bovine serum albumin (BSA) containing 0.5\% Tween-20 in PBS for $45 \mathrm{~min}$. The sections were then incubated overnight at $4^{\circ} \mathrm{C}$ with wellcharacterized goat polyclonal or mouse monoclonal anti-Oct4 antibodies (Santa Cruz Biotechnology, Inc.; final dilution, 1:50). Following the PBS wash, the tissue sections were incubated with biotinylated donkey anti-goat or anti-mouse immunoglobulin G (1:100; Jackson ImmunoResearch) for $1 \mathrm{~h}$. Tissue sections were washed in PBS and then incubated in extravidin/peroxidase conjugate (1:500; Sigma, St. Louis, MO, USA) for $1 \mathrm{~h}$. The antigen-antibody binding sites were revealed by using 3,3'-diaminobenzidine tetrahydrochloride (DAB; Sigma).

Some slides with biopsy and cancer tissue sections were processed for double fluorescent labeling using anti-Oct4 anti- 
bodies and lectins. In these cases, the secondary antibody was biotinylated donkey anti-goat or anti-mouse immunoglobulin G. Antigen-antibody binding sites were visualized using avidin conjugated to fluorescein isothiocyanate (FITC) or rhodamine. After washing with PBS, the tissue sections were incubated with fucose-specific Ulex europaeus agglutinin I (UEA) or $N$-acetylD-glucosamine-specific Griffonia simplicifolia II (GSII) lectins conjugated to FITC or rhodamine. The tissue sections were finally washed with PBS and mounted. Negative control slides were prepared in parallel by either omitting the primary antibody, or by replacing it with normal serum or by pre-incubating the primary antibody with the blocking peptide (Oct4).

Quantification of Oct4 expression. Two methods were used to provide a numerical assessment of the Oct 4 expression in immunoperoxidase-labeled tissue sections. These were as follows:

i) Manually using the $\mathrm{x} 40$ magnification of the light microscope, cells were categorized according to the intensity of immunostaining. At least 3 glands with the best longitudinal orientation were examined in each tissue section of biopsies and cancerous tissues. A score of 3 was given for the most intensely brown-stained cells, intermediate brown staining was given a score of 2 and a score of 1 was given for light brown staining. Unstained cells were given a 0 score. The number of labeled cells was multiplied by the corresponding score. To estimate the average level of Oct 4 expression, the total score of the 3 glands was divided by the total number of cells examined. This was termed the 'expression score' and expressed as the mean \pm SEM value. The means of Oct 4 expression scores were compared by using the ANOVA test and Tukey's post hoc test (PASW statistics 18 ). $\mathrm{P}<0.05$ was considered to indicate statistically significant differences.

ii) To automate the quantification, a computerized method was used to estimate the area of immunolabeled cells per image. This digitalized approach also provides another parameter for quantification of Oct4 expression and speeds up the task of sample examination by several orders of magnitude. The Matlab (Matlab; http://www.mathworks.com/products/ matlab/) image processing library was used for manipulating micrographs of different stomach tissue samples. The following steps for identification and measurement of the labeled areas were applied: a) Image resizing: pixel sizes for the images were reduced and unified. b) Artifact removal: some images included small but varying-size dark spots. The pixels below an (empirically determined) intensity level were replaced by pixels representing the average intensity of the image. c) Color intensity enhancement by using the Matlab imadjust function (MathWorks: Documentation, Image Processing Toolbox imresize; http://www.mathworks.com/access/helpdesk/help/ toolbox/images/imresize.html). Application of this function made the images sharper and removed background colors. d) Identification of the Oct4-labeled area by performing red-green-blue color separation and then using different 'threshold' values to identify the 'brown' areas of the images. The percentage of the area labeled by Oct 4 was finally determined and expressed as a mean \pm SEM value. The means of Oct4 labeled areas were compared using the ANOVA test and Tukey's post hoc test. $\mathrm{P}<0.05$ was considered to indicate statistically significant differences.
Protein extraction and western blot analysis. Frozen tissue samples from biopsies and the 3 regions of gastric cancer were homogenized and lysed using the CelLytic ${ }^{\mathrm{TM}}$ NuCLEAR $^{\mathrm{TM}}$ extraction kit (Sigma). Briefly, sample tissues were rinsed in cold PBS and then homogenized in ice-cold hypotonic lysis buffer (10 mM HEPES, pH 7.9, with $1.5 \mathrm{mM} \mathrm{MgCl}_{2}$ and $10 \mathrm{mM} \mathrm{KCl}$ ) containing $0.1 \mathrm{M}$ DTT and protease inhibitor cocktail made of 4-(2-aminoethyl) benzenesulfonyl fluride (AEBSF), pepstatin A, bestatin, leupeptin, aprotinin, and transepoxysuccinyl-L-leucylamido (4-guanidino)-butane. The crude homogenates were centrifuged for $20 \mathrm{~min}$ at $11,000 \mathrm{x} \mathrm{g}$ at $4^{\circ} \mathrm{C}$. The supernatant represented the cytoplasmic fraction. The remaining pellet was re-suspended in an extraction buffer (20 mM HEPES, pH 7.9, with $1.5 \mathrm{mM} \mathrm{MgCl}_{2}, 0.42 \mathrm{M} \mathrm{NaCl}$, $0.2 \mathrm{mM}$ EDTA, $0.1 \mathrm{M}$ DTT and $25 \%$ glycerol) containing protease inhibitor cocktail for $30 \mathrm{~min}$ at $4^{\circ} \mathrm{C}$. To collect the nuclear fractions, the homogenates were centrifuged for $5 \mathrm{~min}$ at $21,000 \mathrm{x} g$ at $4^{\circ} \mathrm{C}$. Cytoplasmic and nuclear fractions of each of the tissue samples were processed for protein estimation using the BioRad assay kit (BioRad Laboratories, Hercules, CA, USA) and the DU-700 spectrophotometer (Beckman).

Equal amounts of protein (5 or $20 \mu \mathrm{g}$ ) of extracted tissue samples were mixed with $5 \mathrm{X}$ Laemmli buffer $(60 \mathrm{mM}$ Tris- $\mathrm{HCl}$, $\mathrm{pH} 6.8,2 \%$ SDS, $10 \%$ glycerol, $5 \% \beta$-mercaptoethanol, and $0.01 \%$ bromophenol blue) and separated by sodium dodecyl sulfate-polyacrylamide gel electrophoresis (SDS-PAGE) using $10 \%$ acrylamide at $150 \mathrm{~V}$ for $90 \mathrm{~min}$. Protein gels were subsequently transblotted on to nitrocellulose membranes (Schleicher $\&$ Schuell BioScience, Dassel, Germany) at $90 \mathrm{~V}$ for $120 \mathrm{~min}$. Non-specific binding was blocked by $5 \%$ non-fat dry milk in PBS for $1 \mathrm{~h}$. The membranes were then washed with PBS containing $0.1 \%$ Tween-20 (PBST). Subsequently, the membranes were probed overnight at $4^{\circ} \mathrm{C}$ with mouse monoclonal Oct4 (Santa Cruz Biotechnology, Santa Cruz, CA, USA) diluted to 1:500 in PBST and 5\% milk. Following the PBST wash, the blots were incubated with horseradish peroxidase-conjugated goat antimouse immunoglobulin G (Jackson ImmunoResearch) diluted 1:10,000 in PBST for $2 \mathrm{~h}$. Immunoreactive proteins in the blots were detected using SuperSignal West Pico chemiluminescent substrate on CL-Xposure Film (Thermo Scientific, Barrington, IL, USA.). To control equal loading of proteins in the different lanes, the blots were probed with mouse monoclonal anti- $\beta$ actin antibody (Santa Cruz).

\section{Results}

Morphological analysis of the pyloric antral tissues obtained from the mucosal biopsies and from the 3 regions of the resected cancerous stomachs revealed progressive epithelial changes, as previously described (12). While some antral mucosal biopsies appeared normal, others were infiltrated with variable numbers of lymphocytes and plasma cells with evidence of mild or severe gastritis. The latter was commonly associated with atrophic changes in the gastric glands and occasionally showed evidence of metaplasia or even dysplasia. The safe resection margin was determined by cancerous tissues that were hyperplastic and were also associated with metaplastic and dysplastic changes. Tissues from the both tumor edge and center were characterized by a massive increase in mucosal thickness and invasive cancer cells. These series of antral mucosal tissues 
Table I. Manual quantification of the expression scores of Oct4 in control, gastritis and cancer tissues.

A, Expression scores of Oct4 in control, gastritis and cancer tissues

\begin{tabular}{|c|c|c|c|}
\hline Tissues & No. of cells examined & Total score & Mean expression score \pm SEM \\
\hline Control & 963 & 812 & $85.4 \pm 6.8$ \\
\hline \multicolumn{4}{|l|}{ Gastritis } \\
\hline Mild & 1,219 & 1,291 & $110.3 \pm 15.8$ \\
\hline Severe & 1,094 & 1,589 & $130.4 \pm 9.7$ \\
\hline All & 2,313 & 2,880 & $122.1 \pm 8.7$ \\
\hline \multicolumn{4}{|l|}{ Cancer } \\
\hline Safe margin & 854 & 1,110 & $129.1 \pm 16.6$ \\
\hline Tumor edge & 823 & 1,218 & $148.9 \pm 18.3$ \\
\hline Tumor center & 814 & 1,248 & $163.5 \pm 23.4$ \\
\hline All & 2,491 & 3,576 & $147.1 \pm 11.0$ \\
\hline
\end{tabular}

$\mathrm{B}$, Significance (P-value) of the differences in Oct4 expression scores between the controls and gastritis tissues and the 3 regions of gastric cancer

\begin{tabular}{|c|c|c|c|c|c|c|c|}
\hline \multirow[b]{2}{*}{ Tissues } & \multicolumn{3}{|c|}{ Gastritis } & \multicolumn{4}{|c|}{ Cancer } \\
\hline & All & Mild & Severe & All & Safe margin & Tumor edge & Tumor center \\
\hline Control & 0.027 & 0.32 & 0.02 & 0.002 & 0.096 & 0.049 & 0.015 \\
\hline \multicolumn{8}{|l|}{ Gastritis } \\
\hline Mild & & & 0.648 & 0.129 & 0.861 & 0.647 & 0.271 \\
\hline Severe & & & & 0.611 & 0.999 & 0.974 & 0.698 \\
\hline All & & & & 0.146 & 0.861 & 0.647 & 0.271 \\
\hline \multicolumn{8}{|l|}{ Cancer } \\
\hline Safe margin & & & & & & 0.984 & 0.776 \\
\hline Tumor edge & & & & & & & 0.93 \\
\hline
\end{tabular}

The differences in octamer-binding transcription factor 4 (Oct4) expression scores were compared using analysis of variance (ANOVA).

with progressive alterations provided material to investigate the expression pattern of Oct4 in the normal pyloric antrum and during multistep carcinogenesis.

Immunohistochemical localization of Oct4 in normal gastric mucosa. In the tissue sections of normal antral biopsies processed for immunofluorescence histochemistry, Oct4 expression was detected in the basal (nuclear) portion of epithelial cells lining the lower gastric pits next to the gland junction (Fig. 2A). A decreasing gradient of Oct4 labeling toward the luminal surface and glandular bottom was noted. When the same section was also probed with UEA lectin which binds to fucose of pit mucous cell lineage, Oct4-labeled cells also became labeled with UEA. Image overlay showed the co-localization of Oct4 and UEA in the same cells, but in different portions; Oct4 was basal and UEA, apical (Fig. 2B).

The localization of Oct4 in normal gastric mucosa was also demonstrated by using the immunoperoxidase technique. A similar pattern of labeling at the bottom of the gastric pits next to the gland junction was revealed (Fig. 2C). Tissue sections were counterstained with PAS which stained mucus in the apical cytoplasm of Oct4-labeled cells.
Microscopic scoring and quantification of the mean Oct4 expression score in all the control biopsies examined $(n=6)$ was $85 \pm 6.8$ (Table I). The percentages of Oct 4 immunolabeled areas were calculated in binary images and found to have a mean value of $2.19 \pm 0.73$ (Table II).

Immunohistochemical localization of Oct4 in biopsies with gastritis. The probing of gastric tissues obtained from patients with mild gastritis revealed a tendency of pronounced Oct4 immunolabeling in the mucus-producing cells in some biopsies. The area labeled with Oct4 was slightly wider than in the controls (Fig. 2D). Counts revealed that in mild gastritis more cells expressed Oct 4 compared to the control tissues. The estimated mean score of Oct 4 expression in the biopsies examined with mild gastritis was $110 \pm 15.8$ (Table I). However, a comparison of the Oct4 expression scores in the control subjects with those seen in patients with mild gastritis showed no significant difference $(\mathrm{P}=0.32)$.

In the cases of severe gastritis, Oct4-immunolabeled cells were found along the gastric pits and even at the luminal surface. Moreover, a few Oct4-labeled cells were seen scattered deep in the gastric glands (Fig. 2E and F). Since GSII lectin bound to 
Table II. Computerized estimation of the areas of Oct4 labeling in control, gastritis and cancer tissues.

A, Areas of Oct4 labeling in control, gastritis and cancer tissues

\begin{tabular}{lccl}
\hline Tissues & $\begin{array}{c}\text { No. of images } \\
\text { examined }\end{array}$ & $\begin{array}{c}\text { \% Labeled } \\
\text { area }\end{array}$ & SEM \\
\hline Control & 6 & 2.19 & 0.73 \\
All gastritis & 12 & 6.46 & 0.650 \\
All cancer & 5 & 16.13 & 1.22 \\
\hline
\end{tabular}

B, Significance (P-value) of the differences between the areas of Oct4 labeling in control, gastritis and cancer tissues

\begin{tabular}{lcr}
\hline Tissues & All gastritis & All cancer \\
\hline Control & 0.009 & $<0.001$ \\
All gastritis & & 0.013 \\
\hline
\end{tabular}

The differences in octamer-binding transcription factor 4 (Oct4) expression scores were compared using analysis of variance (ANOVA).

the gland mucous cells deep in the gastric mucosa (Fig. 3A), the question arose as to whether the Oct4-labeled cells deep in the gland were indeed some of those that also bound to GSII. Double labeling of tissue sections with the antibody and lectin revealed the co-localization of Oct4 in some GSII-labeled gland mucous cells (Fig. 3B).

Measurements showed that the expression score of Oct4 in tissues with severe gastritis $(130 \pm 9.7)$ was significantly increased when compared to the control patients $(\mathrm{P}=0.02)$. There was, however, no significant difference between the Oct4 expression scores comparing mild with severe gastritis (Table I). When the mean Oct4-labeled percentage areas were estimated for all gastritis specimens, they were found to be significantly different from those of the control patients $(\mathrm{P}=0.009$; Table II).

Immunohistochemical localization of Oct4 in gastric cancer tissues. Since the morphological features of gastric cancer tissues obtained from the 3 sites (safe margin, tumor edge and tumor center) were different, the immunolocalization of Oct4 was examined in each of these sites separately. At the safe (resection) margin of cancerous tissues (an area of the gastric mucosa which is several centimeters away from the tumor), areas of intestinal metaplasia were common. In a tissue section of the safe margin, Oct4 expression was seen along the walls of the pits and also in the scattered metaplastic goblet cells and their associated absorptive cells (Fig. 4A). Mucus-secreting cells at the luminal surface were also immunolabeled with anti-Oct4 antibody. In this tissue, mucus not only in pit cells, but also in goblet cells was labeled with UEA lectin (Fig. 4B).

In the dysplastic areas of the safe margin tissues, there was intensified Oct4 expression (Fig. 4C). Double immunofluorescence labeling using anti-Oct 4 and UEA lectin confirmed the presence of Oct 4 and fucose in the dysplastic mucus-secreting cells (Fig. 4D).
In the safe margin, measurements revealed that the Oct4 expression score was $129 \pm 16.6$ (Table I). This value was not significantly different from that obtained from the tissues of the control biopsies and from mild or severe gastritis tissues ( $\mathrm{P}=0.096,0.861$ and 0.999, respectively).

At the tumor edge, Oct4 expression was associated with the mucus-secreting PAS- and UEA-labeled cells located along the hyperplastic pits and the luminal surface (Fig. 4E). In addition, a large number of labeled cells was found deep in the gastric glands. UEA binding was less extensive than in the control or gastritis tissues. The lectin labeling of pit cells tended to be mainly at the supranuclear Golgi area (Fig. 4F). Measurements of Oct4 labeling in the tumor edge revealed that the mean expression score was $149 \pm 18.3$. This value was significantly different from the score of the control tissues, $\mathrm{P}=0.049$ (Table I).

Since the morphological features of the tumor center were variable, this also showed an inconsistent Oct4 expression profile. Tissue sections with evidence of necrosis and loss of surface epithelium did not reveal Oct4-labeled cells. Tissue sections with intact surface epithelium and many groups of invasive cancer cells showed Oct4 expression on the surface cells which were also positive for PAS or UEA staining. Tissue sections with an intact luminal surface and many glandular profiles showed massive labeling with PAS or UEA in the cells (Fig. 4G and $\mathrm{H}$ ).

Measurements of Oct 4 expression in the tumor center areas without necrotic tissues revealed the highest score (163.5 \pm 23.4$)$ which was significantly different from that of the control tissues $(\mathrm{P}=0.015$; Table I). When the percentage of Oct4-labeled areas was estimated in the tissue sections obtained from all 3 tumor areas, these revealed a mean value (16.13), which was significantly different when compared with the control $(\mathrm{P} \leq 0.001)$ and all gastritis tissues $(\mathrm{P}=0.013)$, as shown in Table II.

Western blot analysis of Oct4 expression in gastric mucosal biopsies and cancerous tissues. To confirm the immunohistochemical localization of Oct4 in control gastric mucosal biopsies and the changes observed during the development of gastritis and gastric cancer, some biopsies $(n=6)$ and cancerous tissues $(n=3)$ were frozen and then processed for western blot analysis.

The specificity of the anti-Oct4 antibody was first examined by using 2 different control tissue extracts believed to be adequate sources of gastric stem/progenitor cells and, hence, rich in Oct4 protein. The first positive control was the gastric mucosal tissue of a newborn mouse which was already shown to have plenty of stem/progenitor cells (27). For comparison, an extract of adult mouse gastric mucosa was also used. When equal amounts of proteins of the newborn and adult gastric mucosal homogenates were loaded for SDS-PAGE, transferred onto a nitrocellulose membrane and subsequently incubated with anti-Oct 4 antibody, a protein band was detected for each of the adult and neonatal extracts at approximately $45 \mathrm{kDa}$ (Fig. 5A). As expected, the band intensity (reflecting the amount of protein present) was much higher in the neonatal than the adult protein extracts.

The second positive control was an epithelial cell line which was previously shown to be representative of gastric stem/progenitor cells (28). This cell line was found to express 

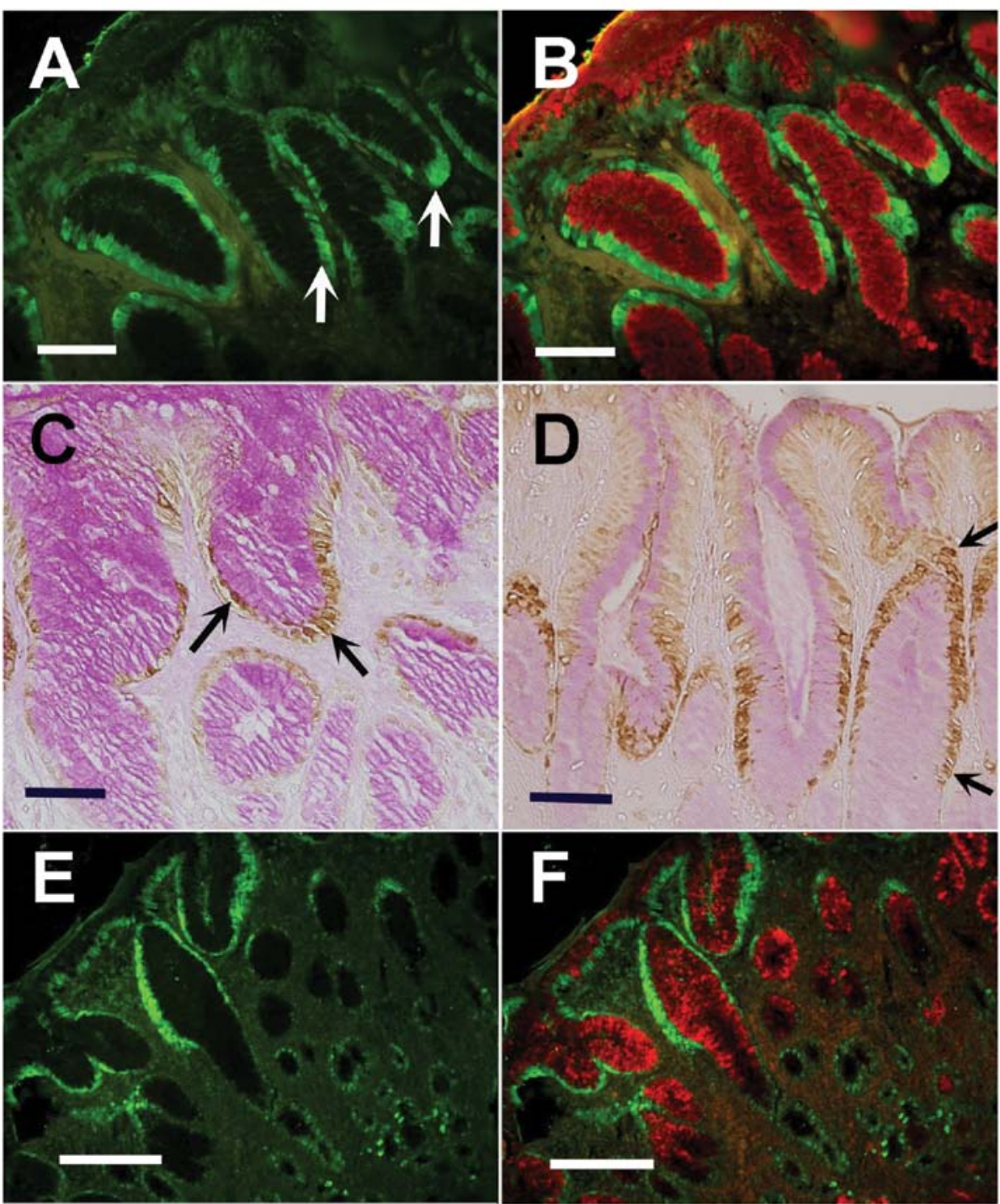

Figure 2. Octamer-binding transcription factor 4 (Oct4) immunohistochemical analysis of pyloric antral tissue sections obtained from biopsies of (A-C) control and (D-F) gastritis individuals. (A) Oct4-labeled cells are seen in the lower portion of the pits (arrows). (B) Overlay with image of labeling with fucose-specific Ulex europaeus agglutinin I (UEA) lectin shows its co-localization with Oct4-labeled cells in the lower portion of the gastric pits next to gland junction. (C) Immunoperoxidase labeling of Oct4 in control gastric mucosa. Oct4-labeled nuclei of periodic acid Schiff (PAS)-positive cells are seen in the lower part of the gastric pit (arrows). (D) Oct4 expression in a mucosal biopsy with mild gastritis. The area labeled with Oct4 appears to be wider (area between the 2 arrows) than in control tissue of (C). (E) Immunofluorescence staining showing Oct4 labeling pattern in a case of severe gastritis. Labeled cells are found along the gastric pits and also scattered deep in the gastric glands (lower right corner). (F) The same section in (E) was incubated with UEA lectin to demonstrate the presence of UEA-labeled mucus in the same Oct4-labeled cells in the pits. Bar, $40 \mu \mathrm{n}$ (A-D) and $80 \mu \mathrm{n}$ (E and F).
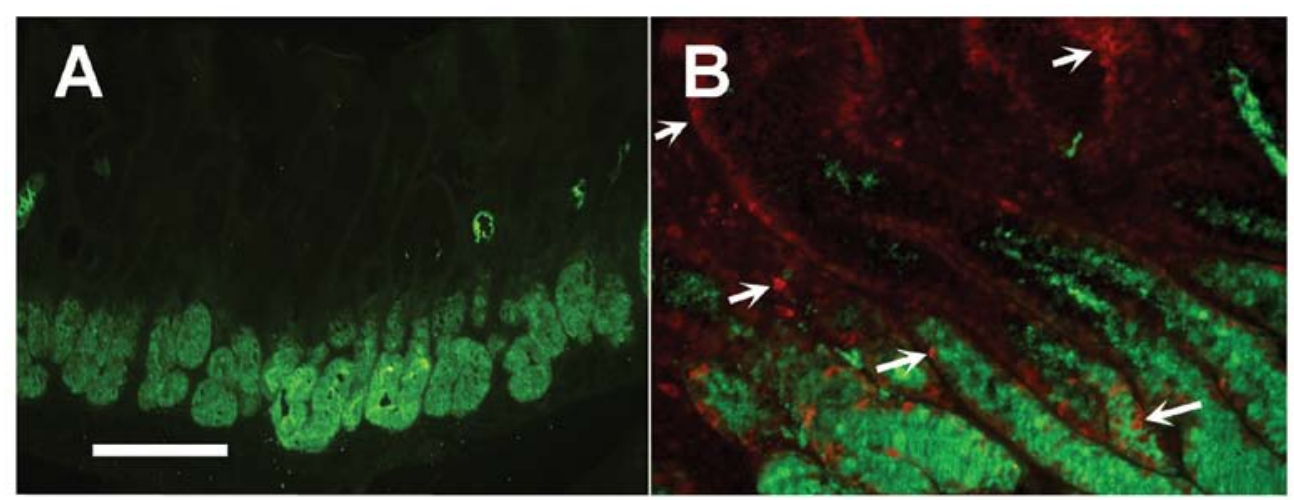

Figure 3. (A) Griffonia simplicifolia II GSII lectin (green) binding in control mucosal tissue. Note that GSII binds to gland mucous cells deep in the mucosa. (B) Double lectin and immunofluorescence staining shows octamer-binding transcription factor 4 Oct4 (red) and GSII (green) labeling patterns in a case of severe gastritis. Oct4-labeled cells are found along the pits and deep in the glands (arrows) where the GSII-labeled mucous cells are present. (A and B) Bar, $80 \mu \mathrm{n}$. 


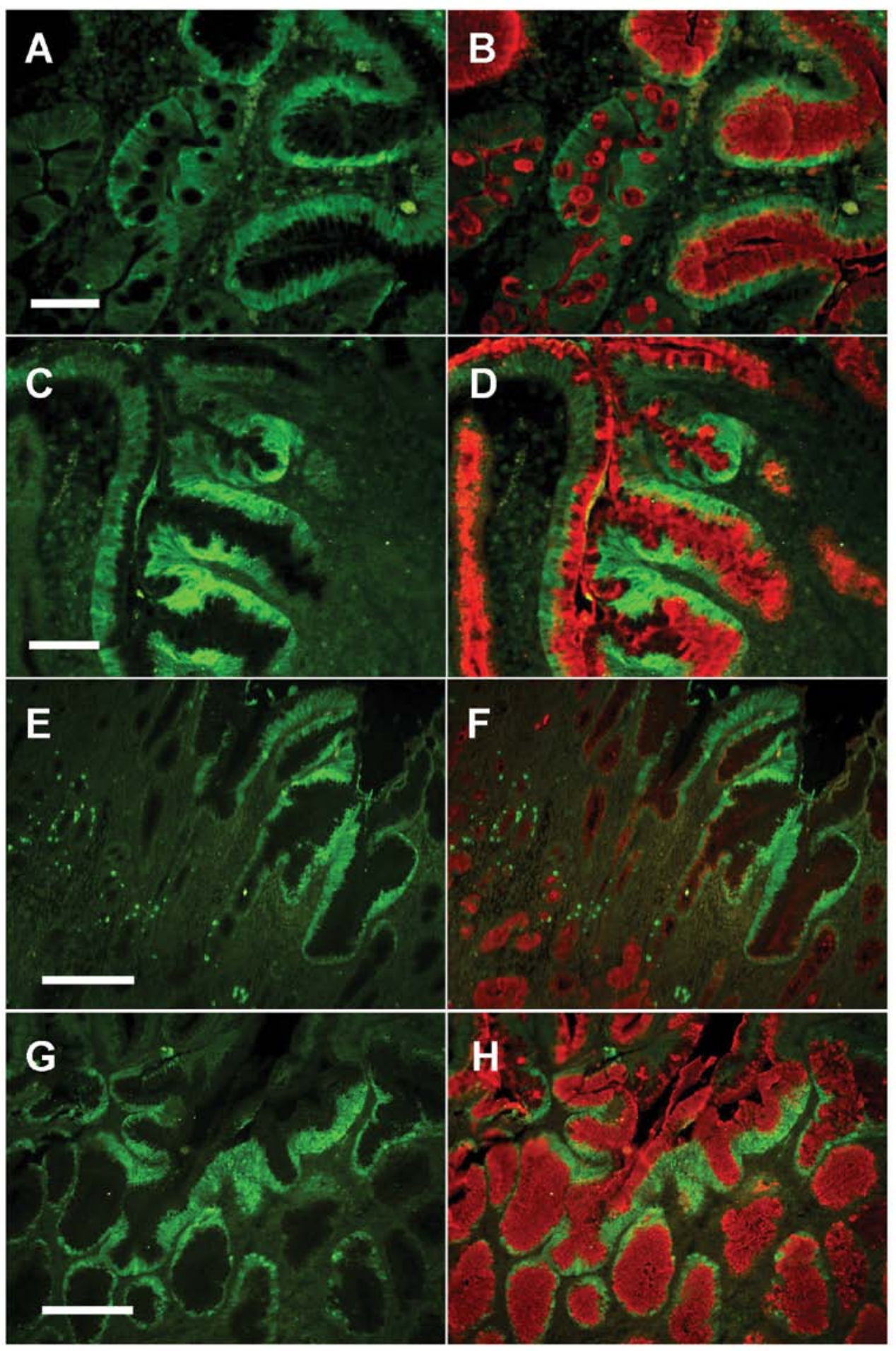

Figure 4. Octamer-binding transcription factor 4 (Oct 4) labeling in cancerous tissues. (A) Oct4 (green) labeling pattern in a case of intestinal metaplasia seen at the safe resection margin of cancer tissue. Oct4 mainly labels the basal portion of the cells along the gastric pits. (B) The same tissue section in (A) was incubated with Ulex europaeus agglutinin I (UEA) lectin to demonstrate the presence of UEA-labeled mucus in the same Oct4-labeled pit cells and also in goblet cells. (C) Anti-Oct4 (green) labeling pattern in a case of dysplasia at the safe margin of cancer tissue. The folded epithelium along the side of a gastric pit expresses Oct4. (D) Anti-Oct4-labeled cells in (C) are UEA lectin-labeled mucous cells. (E) Localization of Oct4 (green) at the edge of cancer tissue. Oct4 expression is evident in the cells lining the hyperplastic glandular profiles and many scattered epithelial cells deep in the gland. (F) The same section in (E) was probed with UEA lectin to label fucose-rich mucous granules in the apical cytoplasm. (G) Localization of Oct4 (green) in the center of cancer tissue. Oct4 expression is evident in the base of the cells lining most of the glandular profiles. $(\mathrm{H})$ The same section in $(\mathrm{G})$ was probed with UEA lectin to label mucous granules in the apical cytoplasm. Bar, $40 \mu \mathrm{n}(\mathrm{A}-\mathrm{D}), 80 \mu \mathrm{n}(\mathrm{E}-\mathrm{H})$

stem cell-specific genes (e.g., Notch3 and DcamK11). A frozen aliquot of this cell line was allowed to thaw and then plated in a culture flask. When 2 different passages of these cells became semi-confluent, they were processed for protein extraction and then separation on a SDS-polyacrylamide gel. Western blot analysis using anti-Oct4 antibodies revealed a single protein 


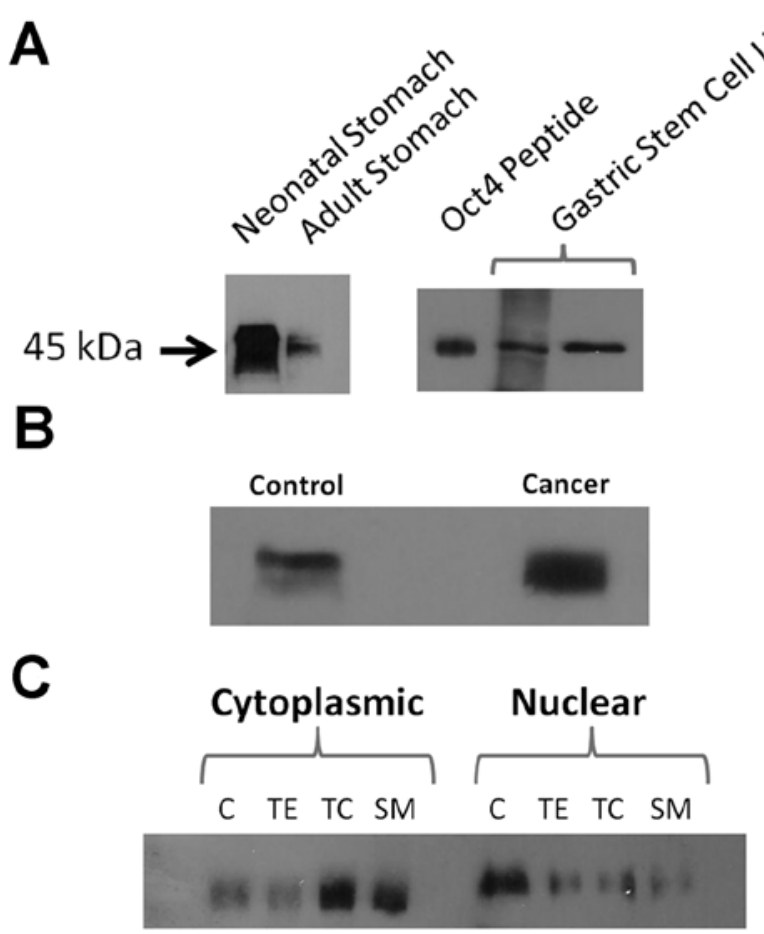

Figure 5. Western blot analysis for the specifity and expression of octamerbinding transcription factor 4 (Oct4). (A) Homogenates of neonatal and adult mouse gastric mucosae (left panel) as well as 2 passages of mouse gastric stem cell lines (right panel) were used as the positive controls of Oct4 expression. Samples were subjected to SDS-PAGE, transblotted onto nitrocellulose membranes and probed with anti-Oct 4 antibody. Oct 4 peptide was loaded in the first plate of the right transblot. (B) Equal amounts $(10 \mu \mathrm{m})$ of the crude homogenates of a control biopsy and a cancer tissue sample were processed for Oct4 probing. Note the difference in the intensity of the bands. (C) Equal amounts $(10 \mu \mathrm{g})$ of cytoplasmic and nuclear fractions obtained from homogenates of a control biopsy and 3 different cancer tissues: tumor edge (TE), tumor center (TC) and safe margin (SM)

band separated at $45 \mathrm{kDa}$, the expected molecular weight of Oct4 (Fig. 5A). The identity of this band was confirmed by pre-incubating the primary antibody with the Oct4 peptide; the band did not develop.

For the human antral tissue samples, to determine whether Oct4 was upregulated in cancerous tissue, the crude homogenates of a control biopsy and tumor tissue (containing equal amounts of proteins) were initially probed for Oct 4 . The results demonstrated the upregulation of Oct 4 expression in cancerous tissue (Fig. 5B).

Since Oct 4 has more than one isoform with debatable subcellular distribution (29-31), it was also essential to clarify its localization using different subcellular fractions. The crude homogenates of human gastric mucosal tissues were processed using the Thermo Scientific Subcellular Protein Fractionation kit. The following subcellular protein fractions were obtained: cytoplasmic, membrane, soluble nuclear, chromatin-bound and cytoskeletal. Equal quantities of proteins from the fractions were loaded on a SDS gel for separation and subsequent transfer to nitrocellulose membranes. Probing with anti-Oct4 antibody revealed that both the cytoplasmic and nuclear fractions were rich in Oct4.

To confirm these findings, another subcellular fractionation protocol was applied using the Sigma CelLytic NuCLEAR extraction kit which has another advantage over the Thermo Scientific protocol. The Sigma procedure is suitable for analysis of small biopsy samples. The homogenized tumor tissues and control biopsies were processed to obtain 2 protein fractions, nuclear and cytoplasmic.

Fractions of the control biopsies were first processed in parallel with those of the cancerous tissues. The results showed an increase in the amount of Oct4 protein in the cancerous tissues as demonstrated in Fig. 5B. The nuclear and cytoplasmic fractions of the control and the cancerous tissues (safe margin, tumor edge and tumor center) were processed separately for western blot analysis using monoclonal antiOct4 antibody. The results revealed a surprising distribution and alteration in Oct4 expression in the various fractions of cancerous tissues. While in the control tissue, the majority of Oct 4 protein was localized in the nuclear fraction, the situation was reversed in the tumor tissues and the majority of Oct4 protein was identified in the cytoplasmic fractions (Fig. 5C). These results suggest that cancer development is associated not only with the upregulation of Oct 4 expression but also with its accumulation in the cytoplasm which could possibly be due to a block in its translocation to the nucleus.

\section{Discussion}

In the present study, the expression profiles of Oct 4 were investigated in an array of human tissues composed of normal, pre-cancerous and cancerous antral mucosae by using both immunohistochemistry and western blot analysis. In addition, the subcellular localization of Oct 4 was examined in different tissues using 2 fractionation protocols from Sigma and Thermo Scientific.

Expression of Oct4 protein. Oct4 is a transcription factor which is prominently expressed in embryonic stem cells and adult stem/progenitor cells anchored in a number of tissues (16-20). The expression of Oct4 is not only detected in embryonic/adult stem cells, but also in various cancerous tissues and cell lines $(20,22-25)$.

During stem cell differentiation, the expression of Oct4 is downregulated. The ectopic expression of Oct4 has been shown to inhibit the differentiation of progenitor cells and promote the dysplastic growth of the gastrointestinal tract and the skin of adult mice (21).

Knowledge in the field of stem cell biology especially of transcription factors responsible for pluripotency, such as Oct4 is rapidly advancing. During the conduct of this study, a brief report was published by Chen $e t$ al on the expression of Oct4 in gastric cancer tissues and in biopsies with atrophic gastritis (32). The authors found that Oct4 is upregulated in gastric cancer and not expressed in adjacent non-cancerous tissues. They further recommended the use of Oct 4 as a biomarker for the diagnosis of gastric cancer. However, the authors demonstrated a single image of Oct 4 immunohistochemical localization in non-cancerous gastric tissue which showed only a small part of the basal region of the gastric mucosa. The authors may have missed the luminal region of the mucosa which we found to be immunolabeled with anti-Oct 4 antibody.

While Chen et al proposed the use of Oct 4 as a possible biomarker for gastric cancer, their study did not provide an 
answer to a number of questions: i) Whether Oct4 is expressed in the normal human antrum, and if so, which cells along the pit-gland unit are involved in the production of Oct4. ii) Whether Oct4 expression is affected during the development of mild gastritis. iii) Clarification as to how this is related to severe gastritis and to $H$. pylori infection. iv) If Oct4 is upregulated in gastric cancer, it should be clarified whether there are any differences in its expression pattern between the safe (resection) margin, tumor edge and tumor center. v) In which part of the cell Oct4 is localized in normal tissues and during cancer development.

Oct4 is expressed in the proliferative zone of the antrum in normal human stomach. In order to answer the question as to whether or not Oct4 is expressed in the normal antrum of the human stomach, and if so, to identify the location of the cells responsible for its expression (isthmus or the bottom of the gland), tissue sections of normal 'control' biopsies were first examined. As it is not usually possible to find normal (healthy) volunteers for endoscopy, we examined biopsies from individuals with upper gastrointestinal symptoms, but with macroscopically normal gastric mucosa. When some of these biopsies were examined microscopically, they had features similar to those of normal control individuals. As regards $H$. pylori, many $(\mathrm{n}=15)$ of these control individuals tested negative, very few $(n=2)$ tested positive and the remainder $(n=8)$ were not tested.

In normal control antral mucosal biopsies, the expression of Oct4 was demonstrated by both immunoperoxidase and immunofluorescence techniques. Oct4 expression was found in the basal portion of PAS-positive cells lining the lower part of the gastric pits at the pit-gland junction (isthmus region). These double PAS-Oct4-positive cells also bind UEA lectin, and therefore, are fucose-rich. Furthermore, some of these Oct4 positive cells also express the proliferating cell nuclear antigen (PCNA) and are hence, proliferative (Fig. 6A and B). Thus, based on their location and proliferative potential, in the normal (control) human stomach, Oct4 is expressed in dividing mucus-producing pre-pit and differentiating pit cells located in the isthmus region of the pit-gland units.

It has been previously shown that in the mouse stomach, pre-pit cells and even differentiating pit cells maintain some capacity of self renewal which gradually decreases as the cells migrate up toward the luminal surface $(7,8)$. In the pyloric antrum of the human stomach, the epithelial lining includes progenitor cells which contain some mucous granules and are capable of self-renewal. These progenitors act as committed stem cells and give rise to the mucus-secreting pit and gland cells (12). It appears that Oct4 is required for the self-renewal of pre-pit cells and also to maintain some capacity of division in differentiating pit cells which gradually decreases with migration, as indicated by the decreasing gradient of Oct 4 expression towards the luminal surface.

To quantify the expression of Oct4 in the tissue sections of normal human stomach, 2 methods were used: manual and computer-based. The former method provided an estimation of the expression score and the computerized images provided a rapid measurement of the percentage of labeled areas. These 2 parameters may be useful in providing complementary ways to investigate the expression of Oct4 in tissue sections.
In the present study, 4 of the control patients with microscopically normal gastric mucosa showed slightly different patterns of Oct 4 expression. Oct4 was localized in both the lower and upper parts of the gastric pits. One possible explanation may be linked to the ingestion by these patients of iron or omeprazole which were found to induce histopathological changes in the gastric mucosa $(33,34)$. Another less likely explanation is that Oct 4 may be normally expressed in certain mature differentiated mucous cells in some individuals. These results are in agreement with those from previous studies which demonstrated Oct 4 expression in fully differentiated peripheral blood mononuclear cells. This finding provides a new perspective challenging the use of Oct4 as a marker specific for stem cells (35). Finally, it should be stated that in these microscopically normal individuals $(n=17)$, we found only $2 H$. pylori-positive cases and they did not reveal any alteration in the pattern of Oct4 expression when compared with the H.pylori-negative cases.

Oct4 expression is altered during the multistep process of gastric carcinogenesis. The development of gastritis was associated with the accumulation of lymphoid cells in the connective tissue between the pit-gland units. These cells were substantially increased in number with the development of severe gastritis and were associated with atrophy of some glands. Adjacent to these atrophied glands, the glandular profiles were lined with poorly differentiated mucous cells. It was therefore not surprising to find a significant increase in Oct4 immunolabeling in the biopsies with evidence of severe gastritis. Some scattered Oct4-labeled cells (possibly the poorly differentiated cells) were observed deep in the mucosa (Figs. 2F and 3B). Thus, during chronic (severe) gastritis there is an enhancement of cell proliferation and pluripotency as evidenced by the upregulation of Oct4 immunolabeling. Quantification by the two different methods confirmed this observation (Tables I and II).

An immunohistochemical analysis of the tissues obtained from the 3 locations of the resected gastric cancer tissues showed an upregulation of Oct4 expression. These findings confirm recently published data (32). Quantifications of immunoprobed tissue sections confirmed these findings particularly when all the cancerous tissues were considered as one group and compared with the controls and the group with gastritis (Tables I and II).

Subcellular localization of Oct4 in normal and cancerous tissues. In the present study, we also attempted to provide an answer to the question regarding Oct4 intracellular localization; we aimed to identify whether Oct4 expression is nuclear or cytoplasmic. There has been some debate regarding this issue $(29,30,36)$. An attempt to examine the detailed immunolabeling of Oct4 in normal control mucosal biopsies showed its localization in the nuclei with little cytoplasmic immunostaining of UEA-labeled cells lining the lower part of the gastric pits (Figs. 2A and 6C). The UAE- and PAS-positive labeling of the apical cytoplasm of these cells indicated that they belong to the mucus-secreting pit cell lineage. Therefore, Oct4 is mostly localized in the nuclei of the mucus-secreting pre-pit cells and their immediate descendents, the differentiating pit cells. 


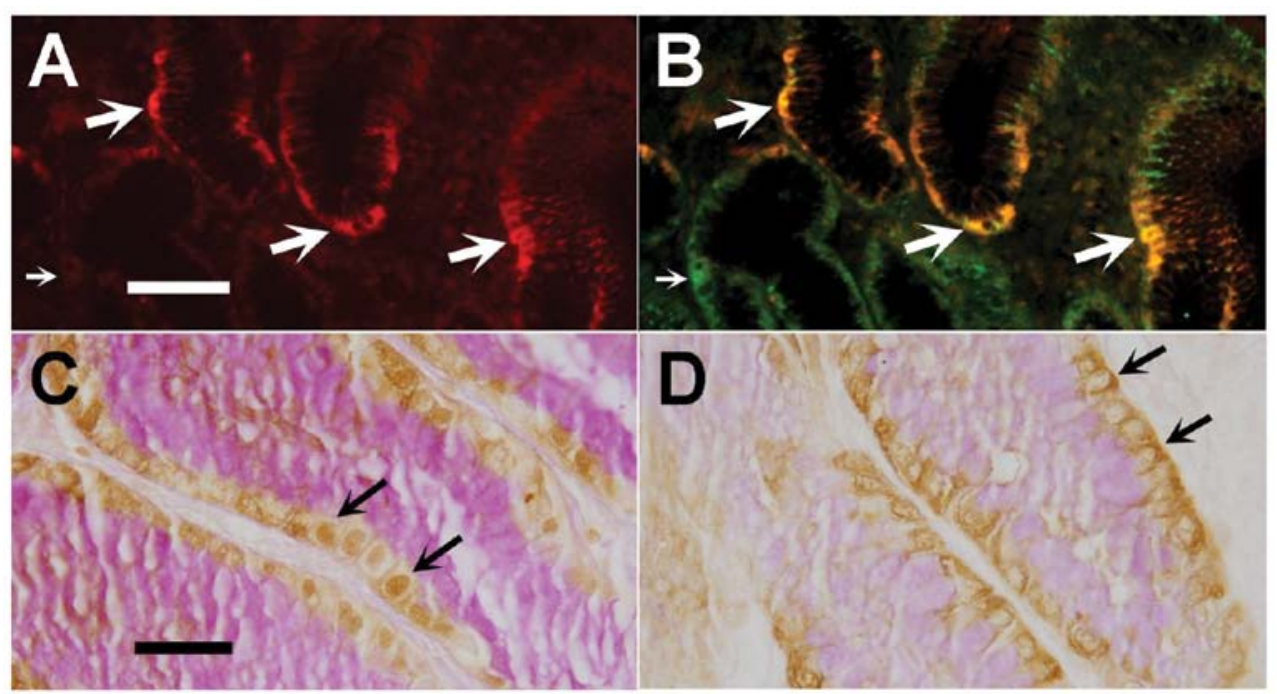

Figure 6. (A and B) Double labeling of octamer-binding transcription factor 4 (Oct4) and proliferating cell nuclear antigen (PCNA) in control gastric mucosal biopsy. (A) Oct4 expression is evident in the cells lining the lower pits [large arrows shown in (A)]. (B) The same section was probed for PCNA and image overlay shows the co-localization of Oct4 in most PCNA-labeled cells. One PCNA-labeled cell seen at the left [small arrow shown in (B)] is not Oct4-labeled. (A and B) Bar, $40 \mu \mathrm{m}$. (C and D) Intracellular localization of Oc4 in the human antrum. (C) In control tissue, Oct4 labeling appears to be mainly nuclear (arrows). (D) In cancerous tissue, Oct4 appears to be mainly cytoplasmic (arrows). (C and D) Bar, $20 \mu \mathrm{m}$.

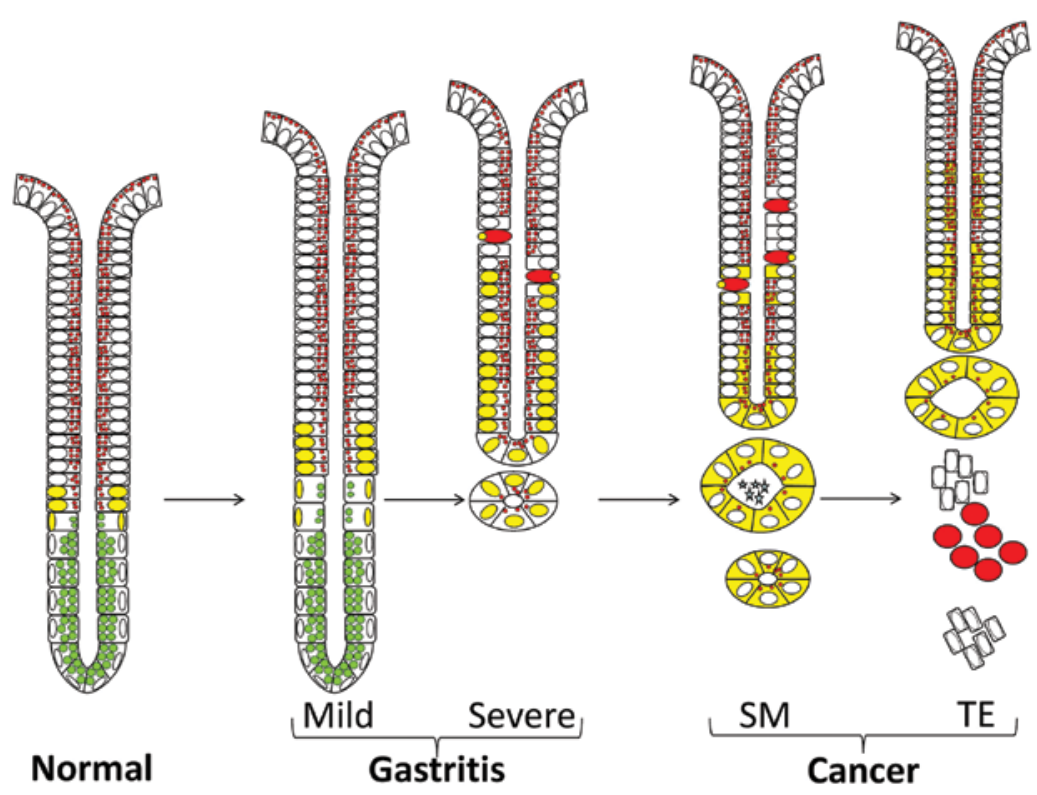

Figure 7. Diagram based on findings from our previous (12) and present studies summarizing the immunolocalization of octamer-binding transcription factor 4 (Oct4) (yellow) in the lower portion of the gastric pit and its alteration during the development of multistep gastric carcinogenesis. SM, safe margin; TE, tumor edge.

Of note, the cancerous tissues did not only show overexpression and intensification of Oct 4 immunostaining, but also showed an alteration in the subcellular localization of Oct4. Microscopic examination revealed that the Oct4 immunolabeling was mostly cytoplasmic (Figs. 6D and 7). This observation was supported by subcellular fractionation and western blot analysis, wherein the majority of Oct4 was localized in the nuclear fraction of normal biopsies, but was mostly localized in the cytoplasmic fraction of cancerous tissues (Fig. 5C). Although the mechanisms that regulate the expression of Oct4 are not yet well understood $(37,38)$, mutation could possibly explain its accumulation in the cytoplasm during carcinogenesis with the inhibition of its translocation into the nucleus.
The data from the present study support the concept of 'cancer stem cells' and the theory of the stem cell origin of cancer $(13,14,38,39)$. Self-renewing progenitor and differentiating cells expressing Oct4 may be the target for cancer initiation and progression, through the induction of symmetrical cell division. The data from the present study also support the view that Oct4 may represent a diagnostic biomarker for gastric cancer. However, further human oncology and mechanistic studies are recommended. Understanding the mechanism by which adult stem or progenitor cells initiate gastric carcinogenesis may well provide greater opportunities for cancer prevention and lead to more effective cancer treatment strategies. 


\section{Acknowledgements}

This study was supported by a research grant from the Terry Fox Fund for Cancer Research.

\section{References}

1. Brenner H, Rothenbacher D and Arndt V: Epidemiology of stomach cancer. Methods Mol Biol 472: 467-477, 2009.

2. Correa P: Is gastric cancer preventable? Gut 53: 1217-1219, 2004.

3. Kushima R, Vieth M, Borchard F, Stolte M, Mukaisho K and Hattori T: Gastric-type well-differentiated adenocarcinoma and pyloric gland adenoma of the stomach. Gastric Cancer 9: 177-184, 2006

4. Correa P, Piazuelo MB and Camargo MC: Etiopathogenesis of gastric cancer. Scand J Surg 95: 218-24, 2006.

5. Fox JG and Wang TC: Inflammation, atrophy, and gastric cancer. J Clin Invest 117: 60-69, 2007.

6. Karam SM: Mouse models demonstrating the role of stem/ progenitor cells in gastric carcinogenesis. Front Biosci 15: 595-603, 2010

7. Lee ER and Leblond CP: Dynamic histology of the antral epithelium in the mouse stomach: II. Ultrastructure and renewal of isthmal cells. Am J Anat 172: 205-224, 1985.

8. Karam SM: Lineage commitment and maturation of epithelia cells in the gut. Front Biosci 4: D286-D298, 1999.

9. Barker N, Huch M, Kujala P van de Wetering M, Snippert HJ, van Es JH, Sato T, Stange DE, Begthel H, van den Born M, Danenberg E, van den Brink S, Korving J, Abo A, Peters PJ, Wright N, Poulsom R and Clevers H: Lgr5(+ve) stem cells drive self-renewal in the stomach and build long-lived gastric units in vitro. Cell Stem Cell 6: 25-36, 2010.

10. Karam SM, Tomasetto C and Rio MC: Trefoil factor 1 is required for the commitment programme of mouse oxyntic epithelial progenitors. Gut 53: 1408-1415, 2004.

11. Karam SM, Tomasetto C and Rio MC: Amplification and invasiveness of gastric epithelial progenitors during carcinogenesis in TFF1-knockout mice. Cell Prolif 41: 923-935, 2008.

12. Al-Awadhi H, John R, Al-Marzooqi F, Vincze A, Branicki F and Karam SM: Sequential alterations in gastric biopsies and tumor tissues support the multistep process of carcinogenesis. Histo Histopath 26: 1153-1164, 2011.

13. Trosko JE and Chang CC: Stem cell theory of carcinogenesis Toxicol Lett 49: 283-295, 1989.

14. Sell S: On the stem cell origin of cancer. Am J Pathol 176: 2584-2594, 2010.

15. Katoh M and Katoh M: WNT signaling pathway and stem cell signaling network. Clin Cancer Res 13: 4042-4045, 2007.

16. Okamoto K, Okazawa H, Okuda A, Sakai M, Muramatsu M and Hamada $\mathrm{H}$ : A novel octamer binding transcription factor is differentially expressed in mouse embryonic cells. Cell 60 461-472, 1990

17. Thomas T, Nowka K, Lan L and Derwahl M: Expression of endoderm stem cell markers: evidence for the presence of adult stem cells in human thyroid glands. Thyroid 16: 537-544, 2006.

18. Yu H, Fang D, Kumar SM, Li L, Nguyen TK, Acs G, Herlyn M and $\mathrm{Xu} \mathrm{X}$ : Isolation of a novel population of multipotent adult stem cells from human hair follicles. Am J Pathol 168: 1879-1888, 2006.

19. Sotomayor P, Godoy A, Smith GJ and Huss WJ: Oct4A is expressed by a subpopulation of prostate neuroendocrine cells. Prostate 69: 401-410, 2009.

20. Tai MH, Chang CC, Kiupel M, Webster JD, Olson LK and Trosko JE: Oct4 expression in adult human stem cells: evidence in support of the stem cell theory of carcinogenesis. Carcinogenesis 26: 495-502, 2005
21. Hochedlinger K, Yamada Y, Beard C and Jaenisch R: Ectopic expression of Oct-4 blocks progenitor-cell differentiation and causes dysplasia in epithelial tissues. Cell 121: 465-477, 2005.

22. Looijenga LH, Stoop H, de Leeuw HP, de Gouveia Brazao CA, Gillis AJ, van Roozendaal KE, van Zoelen EJ, Weber RF, Wolffenbuttel KP, van Dekken H, Honecker F, Bokemeyer C, Perlman EJ, Schneider DT, Kononen J, Sauter G and Oosterhuis JW: POU5F1 (OCT3/4) identifies cells with pluripotent potential in human germ cell tumors. Cancer Res 63 : 2244-2250, 2003.

23. Ezeh UI, Turek PJ, Reijo RA and Clark AT: Human embryonic stem cell genes OCT4, NANOG, STELLAR, and GDF3 are expressed in both seminoma and breast carcinoma. Cancer 104: 2255-2265, 2005

24. Iki K and Pour PM: Expression of Oct4, a stem cell marker, in the hamster pancreatic cancer model. Pancreatology 6: 406-413, 2006.

25. Gibbs CP, Kukekov VG, Reith JD, Tchigrinova O, Suslov ON, Scott EW, Ghivizzani SC, Ignatova TN and Steindler DA: Stem-like cells in bone sarcomas: implications for tumorigenesis. Neoplasia 7: 967-976, 2005.

26. Stolte M and Meining A: The updated Sydney system: classification and grading of gastritis as the basis of diagnosis and treatment. Can J Gastroenterol 15: 591-598, 2001

27. Karam SM,Li Q and Gordon JI: Gastric epithelial morphogenesis in normal and transgenic mice. Am J Physiol 272: G1209-G1220, 1997.

28. Farook VS, Alkhalaf $M$ and Karam SM: Establishment of a gastric epithelial progenitor cell line from a transgenic mouse expressing the simian virus 40 large $\mathrm{T}$ antigen gene in the parietal cell lineage. Cell Prolif 41: 310-320, 2008.

29. Liedtke S, Stephan M and Kogler G: Oct4 expression revisited: potential pitfalls for data misinterpretation in stem cell research. Biol Chem 389: 845-850, 2008.

30. Zuk PA: The intracellular distribution of the ES cell totipotent markers OCT4 and Sox 2 in adult stem cells differs dramatically according to commercial antibody used. J Cell Biochem 106: 867-877, 2009.

31. Wang X and Dai J: Concise review: isoforms of OCT4 contribute to the confusing diversity in stem cell biology. Stem Cells 28: 885-893, 2010

32. Chen Z, Xu WR, Qian H, Zhu W, Bu XF, Wang S, Yan YM, Mao F, Gu HB, Cao HL and Xu XJ: Oct4, a novel marker for human gastric cancer. J Surg Oncol 99: 414-419, 2009.

33. Graham DY, Opekun AR, Yamaoka Y, Osato MS and el-Zimaity HM: Early events in proton pump inhibitor-associated exacerbation of corpus gastritis. Aliment Pharmacol Ther 17: 193-200, 2003

34. Ji H and Yardley JH: Iron medication-associated gastric mucosal injury. Arch Pathol Lab Med 128: 821-822, 2004.

35. Zangrossi S, Marabese M, Broggini M, Giordano R, D'Erasmo M, Montelatici E, Intini D, Neri A, Pesce M, Rebulla P and Lazzari L: Oct-4 expression in adult human differentiated cells challenges its role as a pure stem cell marker. Stem Cells 25: 1675-1680, 2007.

36. Asadi MH, Mowla SJ, Fathi F, Aleyasin A, Asadzadeh J and Atlasi Y: OCT4B1, a novel spliced variant of OCT4, is highly expressed in gastric cancer and acts as an antiapoptotic factor. Int J Cancer 128: 2645-2652, 2011

37. Niwa H, Miyazaki J and Smith AG: Quantitative expression of Oct-3/4 defines differentiation, dedifferentiation or self-renewal of ES cells. Nat Genet 24: 372-376, 2000.

38. Ratajczak MZ, Shin DM, Liu R, Marlicz W, Tarnowski M, Ratajczak $\mathbf{J}$ and Kucia M: Epiblast/germ line hypothesis of cancer development revisited: lesson from the presence of Oct- $4^{+}$ cells in adult tissues. Stem Cell Rev 6: 307-316, 2010.

39. Sell S: Cancer and stem cell signaling: a guide to preventive and therapeutic strategies for cancer stem cells. Stem Cell Rev 3: 1-6, 2007. 